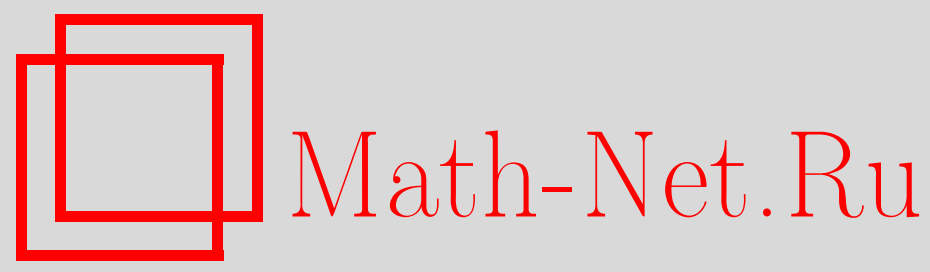

Н. П. Редькин, Об оценках сложности схем из многовходовых функциональных элементов, Дискрет. матем., 2010, том 22, выпуск 1, 50-57

DOI: https://doi.org/10.4213/dm1083

Использование Общероссийского математического портала Math-Net.Ru подразумевает, что вы прочитали и согласны с пользовательским соглашением http://www . mathnet.ru/rus/agreement

Параметры загрузки:

IP : 3.95 .254 .165

26 апреля 2023 г., 06:31:20 


\title{
Об оценках сложности схем из многовходовых функциональных элементов
}

\author{
() 2010 г. Н. П. Редькин
}

\begin{abstract}
В работе представлен метод получения нижних оценок сложности схем из функциональных элементов, основанный на замене рассматриваемого, быть может, достаточно сложного базиса, содержащего многовходовые элементы, на более простой базис, например, из двухвходовых элементов, с последующим использованием уже известных нижних оценок сложности схем в простом базисе. Эффективность предлагаемого метода демонстрируется на конкретных примерах нахождения асимптотики, и, в некоторых случаях, и точного значения для сложности реализации конкретных булевых функций - монотонных симметрических функций и функций с малым числом единиц.

Работа выполнена при финансовой поддержке Российского фонда фундаментальных исследований, проект 08-01-00863, и Программы Президента Российской Федерации поддержки ведущих научных школ, проект НШ 4470.2008.1.
\end{abstract}

\section{1. Введение}

Фундаментальной проблемой математической кибернетики является построение оптимальных, в том или ином смысле, управляющих систем, в частности, построение минимальных схем из функциональных элементов [1] для конкретных булевых функций. При построении минимальных схем обычно приходится решать две задачи: вначале строить подходящие достаточно простые схемы, а затем доказывать минимальность (иногда хотя бы в асимптотике или даже по порядку) этих схем. Первую задачу во многих случаях, представляющих научный и практический интерес, удается решить довольно быстро и легко. Для доказательства утверждений о минимальности, в том числе и об асимптотической минимальности схем из функциональных элементов для конкретных булевых функций, требуется получать достаточно хорошие нижние оценки сложности схем, реализующих заданные функции. Получение таких оценок почти всегда вызывает затруднения, иногда весьма значительные; исключения составляют разве что тривиальные случаи типа нахождения сложности реализации конъюнкции $x_{1} \& \ldots \& x_{n}$ схемами в базисах, содержащих $x \& y$.

Известные к настоящему времени способы получения нижних оценок сложности схем для конкретных булевых функций зачастую предполагают достаточно простые базисы, как правило, содержащие лишь одно- и двухвходовые элементы. Возьмем, скажем, метод “забивания” переменных на входах схем булевыми константами. Этот метод позволил, например, установить сложность реализации линейных булевых функций схемами в базисах $\{x \& y, x \vee y, \bar{x}\},\{x \& y, \bar{x}\},\{x \vee y, \bar{x}\}$ (см. [2]). Основан он на использовании следующих простых свойств схем в базисе $\{x \& y, x \vee y, \bar{x}\}$ (а также и в базисах $\{x \& y, \bar{x}\},\{x \vee y, \bar{x}\}$ ). 
Свойство 1. Пусть в схеме $S$ имеются $n+2$ входов, на которые подаются переменные $x_{1}, x_{2}, \ldots, x_{n}$ и булевь константы 0 и 1 . Если на вход какого-либо элемента схемь $S$ подается булева константа (то есть тождественный нуль или тождественная единица), то этот элемент можно удалить из схемы так, что реализуемая этой схемой функция не изменится.

Свойство 2. Пусть в схеме $S$ имеется $n+2$ входов, на которые подаются переменные $x_{1}, x_{2}, \ldots, x_{n}$ и булевы константы 0 и 1. Тогда если какой-либо элемент схемы $S$ реализует булеву константу, то этот элемент можно удалить из схемь $S$ так, что реализуемая схемой функция не изменится.

Заметим, что изменение соединений элементов в схеме (после того как некоторые элементы будут удалены) всюду считается возможным. Возможно также использование и других (тоже, как правило, достаточно простых) свойств схем.

Представим, что рассматриваются схемы в базисе $\left\{x_{1} \& \ldots \& x_{l}, x_{1} \vee \ldots \vee x_{l}, \bar{x}\right\}$, где $l \geqslant 3$. Очевидно, что схемы в этом базисе по-прежнему обладают вторым свойством, но вот первое свойство в каких-то случаях может и не выполняться (например, если на один вход трехвходового конъюнктора подается константа 1, а на два других входа подаются $x$ и $y$ ). В результате получение нужной нижней оценки сложности схем стандартным методом забивания переменных будет затруднено или даже вообще будет невозможным. Но оказывается, что полученные нижние оценки схем в простых базисах (скажем, из двухвходовых элементов) можно эффективно использовать для получения аналогичных оценок для схем в более сложных базисах. Суть рассматриваемого подхода к получению новых оценок сложности заключается в замене одного (сложного) базиса другим (более простым) базисом.

Пусть $B$ - произвольный базис с положительными весами элементов, а $S$ - схема в базисе $B$. Как обычно, сумму весов всех элементов $S$ будем считать сложностью схемы $S$ и обозначать эту величину будем через $L_{B}(S)$. Для произвольной реализуемой в базисе $B$ булевой функции $f$ положим

$$
L_{B}(f)=\min L_{B}(S)
$$

где минимум берется по всем схемам в базисе $B$, реализующим $f$. Число $L_{B}(f)$ задает (по определению) сложность реализации функции $f$ схемами в базисе $B$; схему $S$ в базисе $B$, реализующую булеву функцию $f$, будем считать минимальной, если $L_{B}(S)=L_{B}(f)$.

Пусть имеются два произвольных конечных базиса $B, B^{*}$, базис $B$ содержит элементы $E_{1}, \ldots, E_{a}$ с весами $P\left(E_{1}\right), \ldots, P\left(E_{a}\right)$, а базис $B^{*}$ содержит элементы $E_{1}^{*}, \ldots, E_{b}^{*}$ с весами $P\left(E_{1}^{*}\right), \ldots, P\left(E_{b}^{*}\right)$ соответственно. Пусть элементам $E_{1}, \ldots, E_{a}$ приписаны соответственно функции $\varphi_{1}, \ldots, \varphi_{a}$, реализуемые этими элементами, а элементам $E_{1}^{*}, \ldots, E_{b}^{*}$ приписаны функции $\varphi_{1}^{*}, \ldots, \varphi_{b}^{*}$.

Справедливо следующее утверждение о соотношении сложностей реализации одной и той же булевой функции схемами из функциональных элементов в разных базисах.

Теорема 1. Пусть булева функиия $f$ реализуема схемой в базисе $B$ и схемой в базисе $B^{*}$, а функции из $B^{*}$ реализуемы схемами в базисе $B$ и выполняются неравенства

$$
L_{B}\left(\varphi_{i}^{*}\right) \leqslant P\left(E_{i}^{*}\right), \quad 1 \leqslant i \leqslant b .
$$

Тогда выполняется неравенство

$$
L_{B^{*}}(f) \geqslant L_{B}(f) .
$$


Доказательство. Пусть задана произвольная булева функция $f$, реализуемая схемами в базисах $B$ и $B^{*}$. Построим для нее минимальную схему $S^{*}$ в базисе $B^{*}$, для которой выполняется условие

$$
L_{B^{*}}\left(S^{*}\right)=L_{B^{*}}(f) .
$$

Воспользуемся условием теоремы и все функции $\varphi_{1}^{*}, \ldots, \varphi_{b}^{*}$ реализуем минимальными схемами (блоками) $S_{1}, \ldots, S_{b}$, построенными в базисе $B$; из (1) для сложностей блоков $S_{1}, \ldots, S_{b}$ следуют оценки

$$
L_{B}\left(S_{i}\right) \leqslant P\left(E_{i}^{*}\right), \quad 1 \leqslant i \leqslant b .
$$

Все элементы в схеме $S^{*}$ заменим отвечающими им блоками (элемент $E_{i}^{*}$ заменяется при этом блоком $\left.S_{i}, 1 \leqslant i \leqslant b\right)$. В результате такой замены вместо схемы $S^{*}$ в базисе $B^{*}$ получим некоторую схему $S$ в базисе $B$, реализующую прежнюю функцию $f$. Из (3) и (4) следует, что $L_{B^{*}}(f) \geqslant L_{B}(S)$, а это означает, что неравенство (2) выполняется. Теорема доказана.

Покажем теперь на конкретных примерах, как с использованием теоремы 1 можно находить точные или асимптотически точные значения сложности реализации индивидуальных булевых функций схемами в базисах из многовходовых элементов.

\section{2. Оценка сложности для монотонных симметрических пороговых функций}

Рассмотрим базис $B^{*}$, содержащий элементы $E_{1}^{*}, E_{2}^{*}, E_{3}^{*}$, реализующие соответственно функции $\varphi_{1}^{*}=x_{1}, \& \ldots \&, x_{l}, \varphi_{2}^{*}=x_{1} \vee \ldots \vee x_{l}, \varphi_{3}^{*}=\bar{x}$, где $l-$ некоторое заданное натуральное число, не меньшее трех, а вес каждого элемента равен единице (то есть сложность схем будет определяться числом элементов в них). В этом базисе для сложности реализации функции

$$
f_{2}^{n}(\tilde{x})=\bigvee_{1 \leqslant i<j \leqslant n} x_{i} x_{j}
$$

справедлива следующая теорема.

Теорема 2. Выполняется асимптотическое равенство

$$
L_{B^{*}}\left(f_{2}^{n}\right) \sim \frac{2 n}{l-1} .
$$

Доказательство. Верхнюю оценку $L_{B^{*}}\left(f_{2}^{n}\right) \lesssim 2 n /(l-1)$ получим, воспользовавшись конструкцией М. И. Гринчука из [3], основанной на представлении $f_{2}^{n}$ в виде

$$
f_{2}^{n}\left(x_{1}, \ldots, x_{n}\right)=f_{2}^{m}\left(a_{1}, \ldots, a_{m}\right) \vee f_{2}^{m}\left(b_{1}, \ldots, b_{m}\right),
$$

где $m=\lceil\sqrt{n}\rceil$, а каждое $a_{i}$ и каждое $b_{i}$ представляет собой дизъюнкцию не более чем $m$ переменных из множества $\left\{x_{1}, \ldots, x_{n}\right\}$. Каждое $a_{i}$ и $b_{i}$ вычисляется с использованием цепочки не более чем из $m /(l-1)$ дизьюнкторов $E_{2}^{*}$; всего $l$-входовых дизъюнкторов потребуется для этого, как нетрудно заметить, асимптотически не более чем $2 n /(l-1)$ 
штук. После этого на достройку схемы, вычисляющей $f_{2}^{n}$ в соответствии с представлением (5), потребуется по порядку не более чем $\sqrt{n}$ элементов (см. первый раздел из [3]). В итоге получается схема, реализующая $f_{2}^{n}$ и содержащая асимптотически не более чем $2 n /(l-1)$ элементов.

Нижнюю оценку получим заменой базиса $B^{*}$ новым базисом $B$, содержащим двухвходовые конъюнктор и дизъюнктор и (одновходовый) инвертор. Вес каждого элемента из $B$ положим равным $1 /(l-1)$; в таком случае условия (неравенства) (1) теоремы 1 будут выполнены.

Согласно теореме 1 из [3] общее число двухвходовых конъюнкторов, двухвходовых дизъюнкторов и инверторов в любой схеме для $f_{2}^{n}$ не меньше чем $2 n-3$; отсюда следует, что сложность любой схемы для $f_{2}^{n}$ в базисе $B$ не меньше чем $(2 n-3) /(l-1)$, то есть

$$
L_{B}\left(f_{2}^{n}\right) \geqslant \frac{2 n-3}{l-1} .
$$

Применяя теорему 1 из неравенств (2) и (6) получаем, что

$$
L_{B *}\left(f_{2}^{n}\right) \gtrsim \frac{2 n}{l-1} .
$$

Теорема доказана.

\section{3. Оценка сложности булевых функций с малым числом единиц}

Рассмотрим класс булевых функций $F_{n, k}$, состоящий из всех тех булевых функций от $n$ переменных, каждая из которых обращается в единицу ровно на $k$ наборах значений переменных. Для класса $F_{n, k}$ Б. И. Фиников установил [4], что если $k=O(\log n)$, то все функции из $F_{n, k}$ реализуемы в классе схем из функциональных элементов (в любом конечном функционально полном базисе) с линейной по $n$ сложностью. Асимптотику для сложности реализации почти всех булевых функций из $F_{n, k}$ в случаях, когда $\log n=o\left(\min \left\{k, 2^{n}-k\right\}\right)$, нашел О. Б. Лупанов [5]; относительно базиса в [5] предполагается лишь то, что он конечный и функционально полный, а положительные веса элементов базиса могут назначаться произвольно. Автором в [6] найдена асимптотика сложности реализации каждой функции из $F_{n, k}$ в случае, когда выполняется условие

$$
1 \leqslant k \leqslant \log n-c \log \log n,
$$

где $c$ - произвольная бо́льшая единицы константа, а базис $B$ содержит элементы, реализующие все булевы функции от двух переменных $x$ и $y$, кроме двух линейных функций $x \oplus y$ и $x \oplus y \oplus 1$ (знак $\oplus$ означает сложение по модулю 2); вес каждого элемента предполагается равным единице.

В рассматриваемом ниже примере предполагается, что условие (7) выполняется, а базис $B^{*}$ содержит $2^{l+1}$ элементов, реализующих конъюнкции $x_{1}^{\sigma_{1}} \& \ldots \& x_{l}^{\sigma_{l}}$ и дизъюнкции $x_{1}^{\sigma_{1}} \vee \ldots \vee x_{l}^{\sigma_{l}}$ для всех булевых наборов $\left(\sigma_{1}, \ldots, \sigma_{l}\right)$ длины $l$, где $l-$ заданное натуральное число, не меньшее трех; вес каждого элемента полагается равным единице (то есть сложность схемы определяется числом элементов в ней).

Напомним некоторые определения из [6]. Пусть $f\left(x_{1}, \ldots, x_{n}\right)$ - произвольная булева функция из $F_{n, k}$, обращающаяся в единицу на наборах $\tilde{\sigma}_{1}, \ldots, \tilde{\sigma}_{k}$, где $\tilde{\sigma}_{i}=\left(\sigma_{i, 1}, \ldots, \sigma_{i, n}\right)$, $i=1, \ldots, k$. Функции $f$ сопоставим $k \times n$ матрицу $M_{f}$, строками которой являются 
наборы $\tilde{\sigma}_{1}, \ldots, \tilde{\sigma}_{k}$, а $j$-й столбец отвечает переменной $x_{j}, j=1, \ldots, n$. Столбцы матрицы $M_{f}$ разобьем на группы одинаковых между собой столбцов; через $M_{\tilde{\tau}}$ обозначим группу столбцов, то есть подматрицу матрицы $M_{f}$, составленную из столбцов, равных $\tilde{\tau}$ (для каких-то $\tilde{\tau}$ группы $M_{\tilde{\tau}}$ могут оказаться пустыми). Непустую группу столбцов $M_{\tilde{\tau}}$ считаем сильной, если она содержит не менее двух столбцов $\tilde{\tau}$ и в этих столбцах имеются как нули, так и единицы; переменные, отвечающие столбцам из сильной группы, также считаем сильными. Число сильных переменных функции $f$ обозначим через $m(f)$.

Теорема 3. Пусть среди переменных функиии $f\left(x_{1}, \ldots, x_{n}\right), f \in F_{n, k}$, имеется $m_{n}$ сильных переменных, а для последовательности $\left\{k_{n}\right\}$ при всех достаточно больших $n$ выполнено условие (7):

$$
1 \leqslant k_{n} \leqslant \log n-c \log \log n,
$$

где с - какая-нибудь бо́льшая единищы константа. Тогда

$$
L_{B^{*}}\left(f\left(x_{1}, \ldots, x_{n}\right)\right) \sim \frac{n+m_{n}}{l-1} .
$$

Доказательство. Верхняя оценка получается конструктивно путем незначительной модификации схемы $S$, представленной в разделе 5 из [6]. В данном случае схему $S$ будем строить с использованием формулы (7) из [6] следующим образом.

По формуле $F_{2,1}$ из [6] построим схему $S_{1}$ (реализующую ту же функцию $f_{1}$, что и формула $F_{2,1}$ из [6]) из $2\left\lceil\left(i_{1}-1\right) /(l-1)\right\rceil+1$ элементов, в которой с помощью $\left\lceil\left(i_{1}-1\right) /(l-1)\right\rceil$ штук $l$-входовых конъюнкторов $x_{1} \& \ldots \& x_{l}$ реализуется конъюнкция $x_{1} \& \ldots \& x_{i_{1}}$, с помощью $\left\lceil\left(i_{1}-1\right) /(l-1)\right\rceil$ элементов $\bar{x}_{1} \& \bar{x}_{2} \& \ldots \& \bar{x}_{l}$ и $x_{1} \& \bar{x}_{2} \& \ldots \& \bar{x}_{l}$ реализуется конъюнкция $\bar{x}_{1} \& \ldots \& \bar{x}_{i_{1}}$, а на выходе дизъюнктора $x_{1} \vee x_{2} \vee \ldots \vee x_{2}$ (его роль играет элемент $x_{1} \vee x_{2} \vee \ldots \vee x_{l}$ с отождествленными 2-м, .., l-м входами) получается требуемая функция $f_{1}$. Аналогично по формулам $F_{2,2}, \ldots, F_{2, r}$ из [6] строятся схемы $S_{2}, \ldots, S_{r}$, реализующие функции $f_{2}, \ldots, f_{r}$. Все вместе схемы $S_{1}, \ldots, S_{r}$ содержат $2\left\lceil\left(i_{1}-1\right) /(l-1)\right\rceil+2\left\lceil\left(i_{2}-i_{1}-1\right) /(l-1)\right\rceil+\ldots+2\left\lceil\left(i_{r}-i_{r-1}-1\right)(l-1)\right\rceil$ элементов; последняя сумма, как не трудно заметить, не превосходит $2 m /(l-1+2 r$.

По формуле $F_{2, r+1}$ из [6] построим схему $S_{r+1}$, состоящую из $\left\lceil j_{s} /(l-1)\right\rceil$ элементов $x_{1} \& \ldots \& x_{l}$ и реализующую ту же функцию, что и формула $F_{2, r+1}$. Схема $S_{r+2}$ из $\left\lceil\left(j_{t}-j_{s}-1\right) /(l-1)\right\rceil$ элементов $\bar{x}_{1} \& \ldots \& \bar{x}_{l}$ и $x_{1} \& \bar{x}_{2} \& \ldots \& \bar{x}_{l}$ отвечает формуле $F_{2, r+2}$ из [6] и реализует ту же функцию $f_{r+2}$, что и формула $F_{2, r+2}$.

Формула $F_{2, r+3}^{\prime}$ из [6] реализует $f_{r+3}$ и представляет собой дизъюнктивную нормальную форму из $k$ конъюнкций, каждая из которых содержит $r+n-i_{r}-j_{t}$ переменных. Формуле $F_{2, r+3}^{\prime}$ сопоставим схему $S_{r+3}$, которая реализует $f_{r+3}$ и содержит $k$ цепочек, реализующих конъюнкции из $F_{2, r+3}^{\prime}$, а также $\lceil(k-1) /(l-1)\rceil$ элементов $x_{1} \vee \ldots \vee x_{l}$, осуществляющих сложение (логическое) конъюнкций в $F_{2, r+3}^{\prime}$; каждая цепочка составляется из $\left\lceil\left(r+n-i_{r}-j_{t}-1\right) /(l-1)\right\rceil$ подходящих элементов $x_{1}^{\sigma_{1}} \& \ldots \& x_{l}^{\sigma_{l}}$. Всего в $S_{r+3}$ окажется $k\left\lceil\left(r+n-i_{r}-j_{t}-1\right) /(l-1)\right\rceil+\lceil(k-1) /(l-1)\rceil$ элементов. Для умножения функций $f_{1}, \ldots, f_{r+3}$, реализуемых на выходах схем $S_{1}, \ldots, S_{r+3}$, можно взять цепочку $S_{r+4}$ из $\left\lceil(r+2 /(l-1)\rceil\right.$ элементов $x_{1} \& \ldots \& x_{l}$; в итоге получим схему $S$, реализующую $f$.

Для сложности полученной схемы $S$ справедлива оценка

$$
\begin{aligned}
& L_{B^{*}}(S) \leqslant \sum_{a=1}^{r+4} L_{B^{*}}\left(S_{a}\right) \leqslant \frac{2 m}{l-1}+ 2 r+\left\lceil\frac{j_{s}}{l-1}\right\rceil+\left\lceil\frac{j_{t}-j_{s}-1}{l-1}\right\rceil \\
&+k\left\lceil\frac{r+n-i_{r}-j_{t}-1}{l-1}\right\rceil+\left\lceil\frac{k-1}{l-1}\right\rceil+\left\lceil\frac{r+2}{l-1}\right\rceil .
\end{aligned}
$$


Отсюда, учитывая неравенство (7) (условие теоремы), соотношение (5) из [6] и равенство $i_{r}=m$, получаем верхнюю оценку для $L_{B}(S)$ (а значит и для $L_{B} *(f)$ )

$$
L_{B^{*}}(S) \lesssim \frac{n+m}{l-1} .
$$

Нижнюю оценку, как и в предыдущем примере, получим при переходе от базиса $B^{*}$ к новому базису $B$, который содержит двухвходовые элементы, реализующие все булевы функции от двух переменных $x$ и $y$, кроме двух функций $(x \oplus y$ и $x \oplus y \oplus 1)$. Вес каждого элемента из $B$ положим равным $1 /(l-1)$; как нетрудно заметить, условия (1) теоремы 1 будут выполнены.

Согласно теореме 1 из [6], общее число элементов из $B$ в любой схеме, реализующей функцию $f\left(x_{1}, \ldots, x_{n}\right)$ из $F_{n, k} \mathrm{c} m$ сильными переменными, асимптотически не меньше $n+m$. Поэтому по теореме 1 сложность любой схемы для $f$ в базисе $B^{*}$ асимптотически не меньше, чем $(n+m) /(l-1)$. Теорема доказана.

\section{4. Об одном семействе классов булевых функций и их сложности реализации}

Пусть $l, m, n$ - натуральные параметры, удовлетворяющие условиям:

(a) $l \geqslant 3$;

(б) $m \leqslant n$;

(в) остаток от деления $m-1$ на $l-1$ не превосходит $l / 2-1$.

Обозначим через $F_{n, 2}^{l, m}$ множество всех тех булевых функций из $F_{n, 2}$, каждая из которых имеет $m$ сильных переменных и при заданном $l \geqslant 3$ для параметров $l, m, n$ выполняются условия (а)-(в).

Пусть $B=\left\{\varphi_{1}, \ldots, \varphi_{8}\right\}-$ множество из восьми булевых функций, каждая из которых есть либо конъюнкция $x^{\alpha} \& y^{\beta}$, либо дизъюнкция $x^{\alpha} \vee y^{\beta}$, где $\alpha, \beta \in\{0,1\}$. При заданном натуральном $l, l \geqslant 3$, через $B^{*}$ обозначим множество всех тех булевых функций, каждая из которых существенно зависит ровно от $l$ переменных $x_{1}, \ldots, x_{l}$ и может быть реализована формулой над $B$ сложности $l-1$; под сложностью формулы $\Phi$ над $B$ понимается число функциональных символов из $B$ в формуле $\Phi$ [7].

Будем рассматривать теперь схемы из функциональных элементов в базисе $B^{*}$ и найдем сложность реализации функций из $F_{n, 2}^{l, m}$ схемами из функциональных элементов в базисе $B^{*}$, полагая вес каждого элемента из $B^{*}$ равным единице.

Теорема 4. Пусть $f(\tilde{x}) \in F_{n, 2}^{l, m}$ и для параметров $l, m, n$ выполняются условия (а)-(в). Тогда

$$
L_{B^{*}}(f)=\left\lceil\frac{n+m-1}{l-1}\right\rceil .
$$

Доказательство. Верхнюю оценку получим конструктивно. Без ограничения общности можем предполагать, что сильными переменными заданной функции $f(\tilde{x})$ являются $x_{1}, \ldots, x_{m}$ (этого, во всяком случае, можно добиться подходящей нумерацией переменных). В таком случае в соответствии с определением класса $F_{n, 2}^{l, m}$ функцию $f$ можно представить в виде

$$
f(\tilde{x})=\left(x_{1}^{\sigma_{1}} \ldots x_{m}^{\sigma_{m}} \vee x_{1}^{\sigma_{1}} \ldots x_{m}^{\sigma_{m}^{-}}\right) x_{m+1}^{\sigma_{m+1}} \ldots x_{n}^{\sigma_{n}} .
$$


Опираясь на представление (8), построим схему $S$ для $f$. Вначале возьмем две цепочки $Z_{1}$ и $Z_{2}$ из элементов базиса $B^{*}$, содержащие по $p$ элементов каждая, где $p=\lfloor(m-1) /(l-1)\rfloor$, и реализующие, соответственно, конъюнкции

$$
\begin{aligned}
& K_{1}=x_{1}^{\sigma_{1}} \ldots x_{p(l-1)+1}^{\sigma_{p(l-1)+1}}, \\
& K_{2}=x_{1}^{\bar{\sigma}_{1}} \ldots x_{p(l-1)+1}^{\bar{\sigma}_{p(l-1)+1}}
\end{aligned}
$$

(если окажется, что $p=0$, то цепочки $Z_{1}$ и $Z_{2}$ в $S$ будут отсутствовать). Добавим к $Z_{1}$, $Z_{2}$ элемент $E$, реализующий

$$
\varphi=\left(K_{1} x_{p(l-1)+2}^{\sigma_{p(l-2)+2}} \& \ldots \& x_{m}^{\sigma_{m}} \vee K_{2} x_{p(l-1)+2}^{\bar{\sigma}_{p(l-2)+2}} \& \ldots \& x_{m}^{\bar{\sigma}_{m}}\right) \& x_{m+1}^{\sigma_{m+1}} \ldots x_{m+q}^{\sigma_{m+q}},
$$

где $q=\min \{n-m, l-2(m-p(l-1))\}$ (если $n=m$, множитель $x_{m+1}^{\sigma_{m+1}} \ldots x_{m+q}^{\sigma_{m+q}}$ в формуле для $\varphi$ отсутствует).

Заметим, что указанное (формально) значение параметра $q$ на самом деле по построению схемы определяется следующим образом. На входы элемента $E$ подаются конъюнкция $K_{1}$ (с выхода цепочки $Z_{1}$ ), переменные $x_{p}(l-1)+2, \ldots, x_{m}$ (с входов схемы), конъюнкция $K_{2}$ и снова переменные $x_{p(l-1)+2}, \ldots, x_{m}-$ в соответствии с формулой для $\varphi$; до этого момента возможность указанного подсоединения элемента $E$ гарантируется условиями (а)-(в), которым удовлетворяют параметры $l, m, n$. Далее, если у элемента $E$ еще остаются незадействованные входы, то на них подаются переменные $x_{m+1}, \ldots, x_{m+q}$. Может оказаться, что на очередной вход элемента $E$ уже будет подана последняя переменная $x_{n}$, а у элемента $E$ все еще остаются незадействованные входы $v_{1}, \ldots, v_{t}$. Тогда на эти входы $v_{1}, \ldots, v_{t}$ дополнительно подается одна и та же последняя переменная $x_{n}$, а в формуле для $\varphi$ в соответствующем месте (по соседству с $x_{n}^{\sigma_{n}}$ ) еще $t$ раз повторяется множитель $x_{n}^{\sigma_{n}}$. При подаче последней переменной $x_{n}$ на входы элемента $E$ построение схемы $S$ на этом заканчивается; нетрудно заметить, что в этом случае $S$ содержит не более $\lceil(n+m-1) /(l-1)\rceil$ элементов.

Рассмотрим другой случай, когда все входы элемента $E$ оказываются задействованными, последней на вход элемента $E$ подана переменная $x_{m+q}, q \geqslant 0$, но $m+q<n$. В этом случае схема дополняется цепочкой $Z$ из последовательно соединенных элементов $E_{1}, \ldots, E_{h}$. Один вход элемента $E_{1}$ соединяется с выходом элемента $E$, а на остальные входы элемента $E_{1}$ подаются переменные $x_{m+q+1}, \ldots, x_{m+q+l-1}$. Один вход элемента $E_{2}$ соединяется с выходом элемента $E_{1}$, а на остальные входы подаются переменные $x_{m+q+l}, \ldots, x_{m+q+2 l-2}$, и так далее. Наконец, один вход элемента $E_{h}$ соединяется с выходом элемента $E_{h-1}$, а на остальные входы элемента $E_{h}$ подаются переменные $x_{m+q+(h-1)(l-1)+1}, \ldots, x_{n}$; при этом хотя бы одна переменная на вход элемента $E_{h}$ подается, а переменная $x_{n}$, возможно, подается на несколько входов (чтобы все входы нижнего в цепочке элемента оказались задействованными). Опять же нетрудно убедиться, что и в этом случае сложность построенной схемы $S$ не будет превышать $\lceil(n+m-1) /(l-1)\rceil$. Таким образом, верхняя оценка установлена.

Нижнюю оценку получим при переходе от базиса $B^{*}$ к базису $B$. Вес каждого элемента из $B$ положим равным $1 /(l-1)$; в этом случае из определения базиса $B^{*}$ видно, что условия (1) теоремы 1 будут выполнены.

Согласно теореме 3 из [6] любая схема над $B$, реализующая функцию $f(\tilde{x})$ из $F_{n, 2}^{l, m}$, содержит не менее чем $n+m-1$ элементов, то есть выполняется неравенство

$$
L_{B}(f) \geqslant \frac{n+m-1}{l-1} .
$$


Отсюда и из соотношения (2) теоремы 1 следует неравенство

$$
L_{B^{*}}(f) \geqslant \frac{n+m-1}{l-1},
$$

из которого с учетом целочисленности $L_{B} *(f)$ окончательно получаем оценку

$$
L_{B^{*}}(f) \geqslant\left\lceil\frac{n+m-1}{l-1}\right\rceil .
$$

Теорема полностью доказана.

\section{Список литературы}

1. Лупанов О. Б., Асимптотические оценки сложности управляющих систем. Изд-во МГУ, Москва, 1984.

2. Редькин Н. П., Доказательство минимальности некоторых схем из функциональных элементов. Проблемы кибернетики (1970) 23, 83-101.

3. Гринчук М. И., О монотонной сложности пороговых функций. Методы дискретного анализа в теории графов и сложности (1992) 52, 41-48.

4. Фиников Б. И., Об одном семействе классов функций алгебры логики и их реализации в класса П-схем. Докл. АН СССР (1957) 115, №2, 247-248.

5. Лупанов О. Б., Об одном подходе к синтезу управляющих систем - принципе локального кодирования. Проблемы кибернетики (1965) 14, 31-110.

6. Редькин Н. П., О сложности булевых функций с малым числом единиц. Дискретная математика (2004) 16, №4, 20-31.

7. Редькин Н. П., Дискретная математика. ЦПИ при мех.-мат. ф-те МГУ, Москва, 2007.

Статья поступила 8.06.2009. Переработанный вариант поступил 16.12.2009. 